\title{
CB1 receptor affects cortical plasticity and response to physiotherapy in multiple sclerosis
}

\section{OPEN}

Francesco Mori, MD,

$\mathrm{PhD}$

Concetta Ljoka, MD,

$\mathrm{PhD}$

Carolina G. Nicoletti, MD

Hajime Kusayanagi, MD

Fabio Buttari, MD

Laura Giordani

Silvia Rossi, MD, PhD

Calogero Foti, MD

Diego Centonze, MD, $\mathrm{PhD}$

Correspondence to

Dr. Centonze:

centonze@uniroma2.it

\section{ABSTRACT}

Objectives: Therapeutic effects of physical therapy in neurologic disorders mostly rely on the promotion of use-dependent synaptic plasticity in damaged neuronal circuits. Genetic differences affecting the efficiency of synaptic plasticity mechanisms could explain why some patients do not respond adequately to the treatment. It is known that physical exercise activates the endocannabinoid system and that stimulation of cannabinoid CB1 receptors (CB1Rs) promotes synaptic plasticity in both rodents and humans. We thus tested whether CB1R genetic variants affect responsiveness to exercise therapy.

Methods: We evaluated the effect of a genetic variant of the CB1R associated with reduced receptor expression (patients with long AAT trinucleotide short tandem repeats in the CNR1 gene) on long-term potentiation (LTP)-like cortical plasticity induced by transcranial magnetic theta burst stimulation (TBS) of the motor cortex and, in parallel, on clinical response to exercise therapy in patients with multiple sclerosis.

Results: We found that patients with long AAT CNR1 repeats do not express TBS-induced LTPlike cortical plasticity and show poor clinical benefit after exercise therapy.

Conclusions: Our results provide the first evidence that genetic differences within the CB1R may influence clinical responses to exercise therapy, and they strengthen the hypothesis that CB1Rs are involved in the regulation of synaptic plasticity and in the control of spasticity in humans. This information might be of great relevance for patient stratification and personalized rehabilitation treatment programs. Neurol Neuroimmunol Neuroinflammation 2014;1:e48; doi: 10.1212/ NXI.0000000000000048

\section{GLOSSARY}

AATn = AAT trinucleotide short tandem repeat; $\mathbf{A M T}=$ active motor threshold; $\mathbf{A N O V A}=$ analysis of variance; $\mathbf{C B 1} \mathbf{R}=$ cannabinoid CB1 receptor; $\mathbf{C S}=$ conditioning stimulus; $\mathbf{D M D}=$ disease-modifying drug; $\mathbf{E A E}=$ experimental autoimmune encephalomyelitis; EDSS = Expanded Disability Status Scale; FDI = first dorsal interosseus muscle; FLAIR = fluid-attenuated inversion recovery; ICF = intracortical facilitation; ISI = interstimulus interval; iTBS = intermittent TBS; $\mathbf{L I C I}=$ long-interval intracortical inhibition; $\mathbf{L T P}=$ long-term synaptic potentiation; $\mathbf{M E P}=$ motor evoked potential; $\mathbf{M S}=$ multiple sclerosis; $\mathbf{N R S}=$ Numerical Rating Scale; $\mathbf{p p}=$ paired-pulse; PT = physical therapy; RMT = resting motor threshold; RRMS = relapsing-remitting MS; SICF = short-interval intracortical facilitation; $\mathbf{S I C I}=$ short-interval intracortical inhibition; TBS = theta burst stimulation; TMS = transcranial magnetic stimulation; TS = test stimulus; TSE = turbo spin echo.

Physical activity increases the efficiency of synaptic transmission ${ }^{1}$ and consistently causes longterm effects on neuronal function and brain structure. ${ }^{2}$ Use-dependent long-term synaptic potentiation (LTP) is considered the molecular substrate for neurorehabilitation, as enhancing synaptic strength in surviving neurons can mitigate the effects of tissue damage by restoring the excitability of neurons that have lost part of their synaptic inputs. ${ }^{3}$ Physical therapy (PT) can reduce motor deficits in disabled patients with multiple sclerosis (MS) with large interindividual differences. ${ }^{4}$

Cannabinoid CB1 receptors (CB1Rs) regulate LTP in animals, ${ }^{5}$ and phytocannabinoids favor transcranial magnetic theta burst stimulation (TBS)-induced LTP in the primary motor cortex

From the Clinica Neurologica (F.M., C.G.N., H.K., F.B., S.R., D.C.), Dipartimento di Medicina dei Sistemi, and Medicina Fisica e Riabilitativa (C.L., L.G., C.F.), Dipartimento di Sanità Pubblica e Biologia Cellulare, Università Tor Vergata, Rome, Italy; and Centro Europeo per la Ricerca sul Cervello (CERC)/Fondazione Santa Lucia (F.M., C.G.N., H.K., F.B., S.R., D.C.), Rome, Italy.

Go to Neurology.org/nn for full disclosures. Funding information and disclosures deemed relevant by the authors, if any, are provided at the end of the article. The Article Processing Charge was paid by Dipartimento di Medicina dei Sistemi, Facoltà di Medicina e Chirurgia, Università degli Studi di Roma Tor Vergata, Rome, Italy.

This is an open access article distributed under the terms of the Creative Commons Attribution-Noncommercial No Derivative 3.0 License, which permits downloading and sharing the work provided it is properly cited. The work cannot be changed in any way or used commercially. 
of patients with MS. ${ }^{6}$ Notably, both TBSinduced $\mathrm{LTP}^{7,8}$ and cannabinoids ${ }^{9}$ had some beneficial effects on spasticity and motor disability in patients with MS, further substantiating the involvement of CB1Rs in the neuroplastic adaptations mediating recovery of function after PT. Accordingly, exercise causes a dramatic upregulation of central CB1R sensitivity, ${ }^{10}$ which contributes to the beneficial clinical effects of exercise in mice with experimental autoimmune encephalomyelitis (EAE), the animal model of MS. ${ }^{11}$

We recently demonstrated that a microsatellite polymorphism of the CB1R-encoding gene (CNR1), an AAT trinucleotide short tandem repeat (AATn) downstream of the translation site, ${ }^{12}$ affects transcription efficacy, as individuals with long-AATn $(\geq 12$ in both alleles) express lower levels of CB1R. ${ }^{13}$ Based on the notion that CB1Rs are involved in the central effects of exercise, we explored whether clinical response variability to PT in MS depends on CB1R function secondary to different genetic background. Our results show that individuals with long-AAT repeats in the CNR1 gene have both defective cortical plasticity in response to intermittent TBS (iTBS) and poor clinical benefits in response to PT.

METHODS Standard protocol approvals, registrations, and patient consents. The study was approved by the institutional review board. Written informed consent was signed by all study participants.

Patients with MS. Thirty Central-Southern Italian patients with relapsing-remitting MS (RRMS) ${ }^{14}$ were enrolled (table) by referral from the MS Center, Neurology Unit, Policlinico Tor Vergata, Rome. Data collection was performed from 2010 to 2012. Sample size was determined on the basis of a previous study. ${ }^{8}$

Patient eligibility was based on the following criteria: (1) clinically definite RRMS, ${ }^{14}$ (2) remitting phase of disease, (3) Expanded Disability Status Scale (EDSS) score between 2 and 7, and (4) presence of spasticity in the lower limbs.

Disease onset was defined as the first episode of focal neurologic dysfunction indicative of MS. All patients were in a remitting phase in the 3 months preceding recruitment and during the study. Remission was defined as the absence of new or recurrent neurologic symptoms not associated with fever or infection lasting for at least 24 hours.

All patients started disease-modifying drugs (DMDs) at the time of diagnosis. First-line treatment prescribed was glatiramer acetate (20 mg SC daily), interferon $\beta-1 \mathrm{a}$ ( $44 \mu \mathrm{g}$ SC 3 times weekly), interferon $\beta$-1a (30 $\mu \mathrm{g} \mathrm{IM})$, or interferon $\beta$-1b $(250 \mu \mathrm{g}$ SC every other day). Mitoxantrone $\left(12 \mathrm{mg} / \mathrm{m}^{2} \mathrm{IV}\right.$ every 3 months with a lifetime maximum of $140 \mathrm{mg} / \mathrm{m}^{2}$ ) or natalizumab (300 mg IV every 4 weeks) was prescribed as second-line treatment.

Determination of AAT repeats in the CNR1 gene. Samples of peripheral blood from recruited patients were collected in BD Vacutainer tubes with EDTA (Beckton Dickinson, Franklin Lakes, NJ). Genomic DNA was isolated and extracted from human whole blood $(200 \mu \mathrm{L})$ using a MagNA Pure LC DNA Isolation Kit and automated extractor (Roche Diagnostics GmbH, Mannheim, Germany) following manufacturer's instructions. One hundred fifty ng of genomic DNA was used to amplify the CNR1 region, including the AAT repeats through PCR. PCR was carried out in a $25 \mu \mathrm{l}$ volume containing polymerase buffer, $1 \mathrm{mM} \mathrm{MgCl}, 0.2 \mathrm{mM}$ of each dNTP, 0.5 pmoles of each primer (sense: 5'-CACCCCTGGGCTGTAAAATA-3'; antisense: 5'-GTTGCAGTGAGCCAAGATCA-3'), and 1.5 U Taq DNA polymerase (Invitrogen, Madison, WI). Denaturation was achieved through a first step of 5 minutes at $94^{\circ} \mathrm{C}$ followed by 35 cycles of 45 seconds at $95^{\circ} \mathrm{C}$, annealing for 1.5 minutes at $58^{\circ} \mathrm{C}$ and elongation for 1 minute at $72^{\circ} \mathrm{C}$, and final elongation for 7 minutes at $72^{\circ} \mathrm{C}$. For sequencing analysis, $10 \mathrm{ng}$ of PCR products Agencourt AMPure PCR Purification kit (Agencourt Bioscience Corporation, Beverly, MA), 0.5 pmoles of the sequencing primer (5'-ACCTCCACCCACAAATCAAA-3'), and the ABI PRISM BigDye Terminator v3.1 Ready Reaction Cycle Sequencing Kit (Applied Biosystems, Foster City, CA) were used. Sequencing reactions initiated with 1 minute

Table Clinico-demographic characteristics of the studied sample

\begin{tabular}{|c|c|c|c|c|c|c|c|c|c|c|c|}
\hline $\begin{array}{l}\text { CNR1 } \\
\text { AATn }\end{array}$ & Sex & Age, y & $\begin{array}{l}\text { Disease } \\
\text { duration, y }\end{array}$ & DMD & LL & \multicolumn{2}{|l|}{ EDSS } & \multicolumn{2}{|l|}{ EDSS-PFS } & \multicolumn{2}{|l|}{ NRS } \\
\hline Short ${ }^{a}$ & $8 \mathrm{M}, 7 \mathrm{~F}$ & $45 \pm 10$ & $8.3 \pm 5.0$ & $\begin{array}{l}4 \text { GA } \\
4 \text { IFN- } \beta-1 a \\
5 \text { IFN- }-1 b \\
1 \text { natalizumab } \\
1 \text { mitoxantrone }\end{array}$ & $7,054 \pm 6,956$ & $4.5 \pm 1.9$ & $4.0 \pm 1.9$ & $3.0 \pm 0.4$ & $1.0 \pm 0.5$ & $6.4 \pm 0.8$ & $4.1 \pm 0.9$ \\
\hline
\end{tabular}

Abbreviations: AATn = AAT trinucleotide short tandem repeat; DMD = disease-modifying drug; EDSS = Expanded Disability Status Scale; GA $=$ glatiramer acetate; IFN = interferon; LL = lesion load; NRS = Numerical Rating Scale (spasticity); PFS = pyramidal functional system subscore.

Values represent median \pm SD for EDSS and EDSS-PFS, and mean \pm SD for age, disease duration, lesion load, and NRS.

${ }^{\text {a }}$ Short $=$ patients carrying at least 1 allele with CNR1 AATn $\leq 11$

${ }^{\mathrm{b}}$ Long $=$ patients carrying both alleles with CNR1 AATn $\geq 12$. 
denaturation at $96^{\circ} \mathrm{C}$ and proceeded with 40 cycles of 10 seconds at $96^{\circ} \mathrm{C}, 5$ seconds at $50^{\circ} \mathrm{C}$, and 4 minutes at $60^{\circ} \mathrm{C}$. Product of sequencing was purified through a CleanSEQ dye terminal removal kit (Agencourt Bioscience Corporation) and run on the Applied Biosystems 3730 DNA Analyzer Instrument (Applied Biosystems), complying with manufacturer's instructions. The number of AAT repeats was counted on the resulting electropherograms.

Disability assessment. All patients were clinically assessed by expert neurologists who were blinded to laboratory and neurophysiology results. EDSS score and a 0-10 Numerical Rating Scale (NRS) score assessing self-reported severity of spasticity ${ }^{9}$ were recorded in all patients with MS before the first and immediately after the last PT session.

MRI acquisition and analysis. 3T MRI scans consisted of a dual-echo turbo spin echo (TSE), a fluid-attenuated inversion recovery (FLAIR), and precontrast and postcontrast T1weighted spin echo images. All images were acquired in the axial orientation with 3-mm-thick contiguous slices. T2 hyperintense lesions were determined by consensus of 2 investigators on proton density-weighted short echo TSE images. Lesions were outlined through semiautomated local thresholding contouring software (Jim 4.0, Xinapse System, Leicester, UK). Confidence in lesion identification was increased using FLAIR and T2-weighted scans as a reference. Presence of lesions within the brainstem, corpus callosum, posterior fossa, periventricular, and spinal cord was recorded for each patient to evaluate lesion distribution.

Transcranial magnetic stimulation. LTP can be measured in patients through transcranial magnetic stimulation (TMS). TMS can activate neurons in a focal region of the cerebral cortex through electromagnetic induction. Delivered repetitively on the motor cortex, TMS can induce plastic modifications of cortical excitability that can be measured by recording the motor evoked potentials (MEPs) from the muscles represented within the stimulated region. An increase or decrease of the MEP amplitude lasting after the end of repetitive TMS indicates that LTPlike or long-term depression-like plastic changes occurred, respectively.

Electromyographic traces were recorded from the right first dorsal interosseus muscle (FDI) with surface electrodes, amplified (Digitimer, Hertfordshire, UK), sampled at $5 \mathrm{kHz}, 20 \mathrm{~Hz}-2 \mathrm{kHz}$ bandpassed, and stored for offline analysis.

MEPs were evoked through a Magstim $200^{2}$ magnetic stimulator (Magstim Company, Whitland, Wales, UK) with a 70-mm diameter figure-of-eight coil. Coil was positioned tangentially to the scalp over the left hemisphere to evoke MEPs from the right FDI ("hot spot"), the handle pointing posterolaterally at $45^{\circ}$.

For iTBS, a Magstim Rapid ${ }^{2}$ delivered 20 cycles of 10 bursts repeated at $5 \mathrm{~Hz}$ and separated by 8 -second pauses, with each burst composed of a triplet of TMS pulses at $50 \mathrm{~Hz}$. Stimulation intensity was $80 \%$ of the active motor threshold (AMT). AMT was defined as the minimum stimulation intensity required to evoke at least $5 \mathrm{MEP}, \sim 200 \mu \mathrm{V}$ peak-to-peak amplitude out of 10 pulses during FDI's voluntary contraction (10\% of maximal). Immediately before iTBS and at 2 different time points $(0$ and 15 minutes) after the end of iTBS, we collected 35 MEPs evoked by a test stimulus (TS) with a stimulation intensity set to induce a stable MEP of approximately $1 \mathrm{mV}$ peak-to-peak amplitude in the relaxed FDI at baseline. MEP amplitudes were averaged at each time point and normalized to the mean baseline amplitude.

Short-interval intracortical inhibition (SICI) and intracortical facilitation (ICF) $)^{15}$ were tested using paired-pulse (pp) TMS with a conditioning stimulus (CS) at $80 \%$ AMT intensity, preceding the TS. ${ }^{15}$ Five conditions were randomly presented: TS alone and 4 pp conditions with CS preceding TS at 1 of 4 different interstimulus intervals (ISIs) $(2,3,10$, and $15 \mathrm{msec})$.

Short-interval intracortical facilitation (SICF) was tested using a CS at $90 \%$ of resting motor threshold (RMT) intensity. Six conditions were randomly presented: TS alone and 5 conditions with TS preceding CS at 1 of 5 different ISIs (1.5, 2.1, $2.7,3.7$, and $4.5 \mathrm{msec}){ }^{16}$

Long-interval intracortical inhibition (LICI), mediated by $\mathrm{GABA}_{\mathrm{B}},{ }^{17}$ was tested through a CS at $120 \%$ RMT intensity. Two conditions were presented in a random order: TS alone and CS preceding TS at an ISI of $100 \mathrm{msec}$. For each experimental condition 10 responses were collected. MEP changes at each ISI were expressed as percentage of the mean unconditioned MEP amplitude.

Physical therapy. Two to 4 weeks after TMS evaluation, patients started the therapeutic exercise program at the Physical Rehabilitation Department of the University Hospital Policlinico Tor Vergata in Rome. Exercises were performed on a daily basis for 2 weeks and consisted of 1 hour on land followed by another hour in $28-30^{\circ} \mathrm{C}$ water in a $150-\mathrm{cm}$ wide pool, as in a previous study. ${ }^{8,18}$ The program was devised and coordinated by a physician specializing in physical medicine and rehabilitation and consisted of both passive and active therapeutic exercises specifically aimed at restoring or maintaining muscular flexibility, range of motion, balance, coordination of movements, postural passages and transfers, and ambulation. Different types of therapeutic exercises were scheduled according to the individual disability status and administered by qualified physiotherapists. The exercises consisted of (1) repetition of different movements (i.e., tips and heels, $90^{\circ}$ flexed hips and knees) for ambulation and stair climbing; (2) repetition of crossed patterns of movements for coordination; (3) postural reactions while standing with eyes open and closed, including the use of oscillatory boards for balance; (4) strengthening lower limb antigravitary muscles (i.e., gluteus minor and major, quadriceps femoralis); and (5) low-intensity and longduration static stretching of iliopsoas, rectus femoralis, hamstrings, triceps surae, and lumbar spinal muscles for muscular flexibility and range of motion.

During each session patients performed either 2 or 3 sets per exercise type, with each set consisting of about 15 repetitions. Intensity of exercise was graded on the level of disability. Compensative pauses of time were included in relation to each patient's tolerance to the exercise regimen, for proper restoring.

Data analysis. As in previous studies, ${ }^{13,19}$ patients with MS were grouped according to the number of AAT repeats of the CNRI gene. In the short-AATn group patients had 1 or 2 alleles with $\leq 11$ repeats of AAT triplets, and in the long-AATn group patients had 2 alleles with $\geq 12$ repeats. Student $t$ test, MannWhitney test, Fisher exact test, and repeated-measures analysis of variance (ANOVA) were used to analyze differences between groups, as appropriate. Type of DMD was entered as a covariate because of its effect as a potential confounder. Duncan post hoc correction was performed. Correlation between EDSS and TMS parameters was explored through regression analysis. Data are presented as mean \pm SEM. $p<$ 0.05 was considered significant.

RESULTS The experimental procedures were welltolerated by all study participants and no adverse reactions were reported. Long- and short-AATn CNR1 patients showed no clinico-demographic 
differences and no differences in MRI lesion load (table) and distribution (data not shown) at baseline.

CNR1 (AAT)n influences clinical response to PT. For EDSS, repeated-measures ANOVA with time (baseline and post-PT) as within-subjects and group (short- and long-AATn) as between-subjects main factors showed an effect of time $(d f=1, F=6.6$, $p<0.01)$ and time-group interaction $(d f=1, F=$ 24.8, $p<0.01$ ). Post hoc contrasts revealed lower EDSS in short-AATn post-PT. Pyramidal functional system EDSS subscore showed an effect of time $(d f=1, F=35.9, p<0.001)$ and time-group interaction $(d f=1, F=37.8, p<0.001)$, with a lower EDSS pyramidal subscore in the short-AATn group post-PT. No differences emerged for the other EDSS functional system subscores (visual, brainstem, cerebellar, sensory, bowel and bladder, and cerebral).

Accordingly, the spasticity NRS showed an effect of time $(d f=1, F=4.2, p<0.05)$ and timegroup interaction $(d f=1, F=6.4, p<0.05)$, with NRS lower in the short-AATn group post-PT (figure 1). DMD, entered as a covariate, had no effect on clinical response to PT.

CNR1 (AAT)n influences cortical plasticity. For iTBS, repeated-measures ANOVA with time (baseline, 0, and 15 minutes post-iTBS) as within-subjects and group (short- and long-AATn) as between-subjects main factors showed an effect of time (mean \pm SE $=$ $1.16 \pm 0.06$ at 0 and $1.29 \pm 0.09$ at 15 minutes postiTBS; $d f=1.78, F=4.38, p<0.05$ ), group (mean \pm $\mathrm{SE}=1.26 \pm 0.10[$ short-AATn] and $1.05 \pm 0.09$ [long-AATn] at 0 , and $1.39 \pm 0.16$ [short-AATn] and $1.13 \pm 0.20$ [long-AATn] at 15 minutes post-iTBS; $d f=1, F=3.64, p<0.05)$, and time-group interaction $(d f=1.78, F=6.43, p<0.05)$. Post

Figure 1 Role of CNR1 AATn in the clinical response to exercise therapy
A

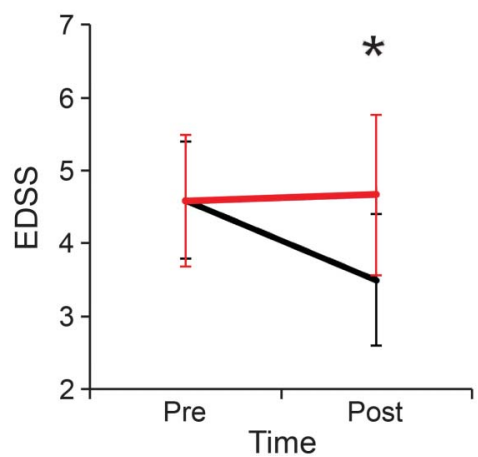

B

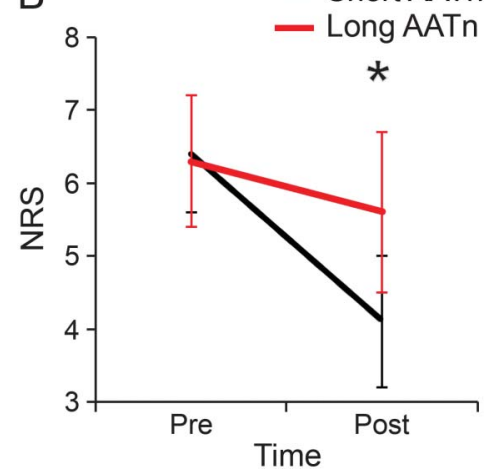

After the 2-week physical therapy program, patients carrying a short number of AATn repeats (AATn $\leq 11$ in at least 1 allele) show a better clinical response in both EDSS (A) and spasticity NRS (B). * $p<0.05$; error bars indicate SD. AATn $=$ AAT trinucleotide short tandem repeat; EDSS $=$ Expanded Disability Status Scale; NRS = Numerical Rating Scale. hoc analysis revealed increased iTBS in short-AATn at 15 minutes.

Conversely, an effect of ISI but not of group or ISI-group interaction emerged for SICI $(d f=2$, $F=8.96, p<0.001)$, ICF $(d f=2, F=3.28$, $p<0.05)$, SICF $(d f=1.97, F=20.43, p<$ $0.001)$, and LICI $(d f=1, F=76.88, p<0.001)$ (figure 2). Student $t$ test did not reveal any betweengroup difference in baseline MEP amplitudes (shortAATn $1.46 \pm 0.18 \mathrm{mV}$ vs long-AATn $1.36 \pm 0.22$ $\mathrm{mV}$ ), RMT (short-AATn $46.7 \pm 8.2$ vs long-AATn $47.2 \pm 8.8$ ), AMT (short-AATn $33.2 \pm 8.2$ vs longAATn $36.2 \pm 8.5$ ), and MEP latency (short-AATn $22.8 \pm 3.3 \mathrm{msec}$ vs long-AATn $22.9 \pm 3.0 \mathrm{msec}$, all $p>0.1)$. DMD, entered as a covariate, had no effect on iTBS, SICI, ICF, SICF, or LICI.

No correlation emerged between the EDSS score measured at baseline and iTBS, SICI, ICF, SICF, or LICI.

DISCUSSION In the present study we observed that patients with MS carrying a genetic variant of the $\mathrm{CB} 1 \mathrm{R}$ associated with reduced receptor expression are refractory to the effects of PT on motor function, and in parallel we found that motor cortex LTP is defective in these patients.

After brain damage, surviving neurons undergo plastic functional reorganization to minimize the clinical manifestation of the lesion. PT boosts this compensatory plasticity, increasing activity-dependent synaptic potentiation and subsequent anatomical reorganization and driving axonal sprouting and synaptogenesis. ${ }^{20}$ In humans, cortical representation of the muscles involved in motor training enlarges and the activation threshold decreases. ${ }^{21}$

LTP is considered the synaptic underpinning of exercise-induced plasticity, as this form of activitydependent plasticity makes synapses more responsive to future stimulations and may thus potentially compensate for the loss of inputs caused by neuronal damage on surviving deafferented neurons. Indeed, both in vitro LTP and exercise cause dendritic growth and new synapse formation, ${ }^{20}$ stimulating similar molecular pathways.

Exercise increases endocannabinoid signaling in humans ${ }^{22}$ and attenuates clinical manifestations of experimental $\mathrm{MS}^{11}$ through increased $\mathrm{CB} 1 \mathrm{R}$ sensitivity. ${ }^{10}$ In contrast, genetic deletion of CB1R is associated with decreased improvement of motor performance induced by exercise ${ }^{23}$ and defective LTP $^{5}$ in mice. Moreover, the CB1R seems to be involved in the control of spasticity both in $\mathrm{EAE}^{24}$ and in patients with $\mathrm{MS}^{9,25}$ even though cannabinoid treatment showed mixed results, as several trials reported subjective benefit from patients whereas blinded assessments by investigators failed to show 
Figure 2 Role of CNR1 AATn in the regulation of cortical excitability
A. ITBS

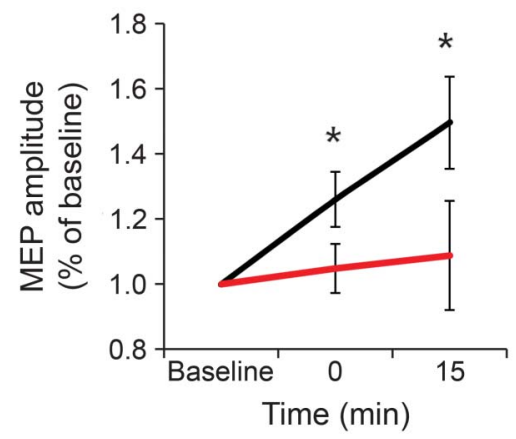

C. LICI

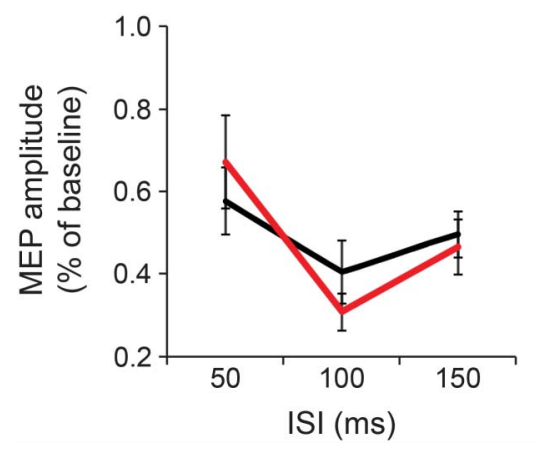

B. SICI \& ICF

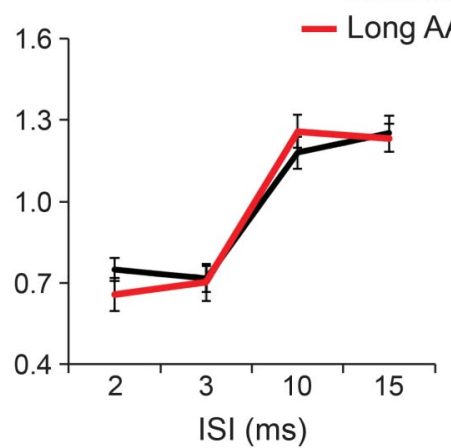

D. SICF

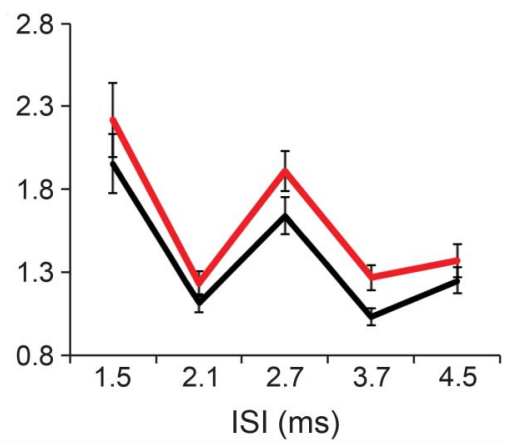

Multiple sclerosis patients with a long number of AATn repeats (AATn $\geq 12$ on both alleles) on the CNR1 gene show defective LTP response to iTBS in the motor cortex. In contrast, patients with a short number of AATn repeats (AATn $\leq 11$ in at least 1 allele) show a preserved LTP response after iTBS (A). No differences were found between short- and longAATn carriers in the SICI/ICF (B), LICI (C), and SICF (D) paired-pulse TMS protocols. *p < 0.05; error bars indicate SEM. AATn = AAT trinucleotide short tandem repeat; ICF = intracortical facilitation; ISI = interstimulus interval; iTBS = intermittent theta burst stimulation; $\mathrm{LICl}=$ long-interval intracortical inhibition; LTP = long-term synaptic potentiation; MEP = motor evoked potential; SICF = short-interval intracortical facilitation; $\mathrm{SICI}=$ short-interval intracortical inhibition; TMS = transcranial magnetic stimulation.

objective changes in spasticity. Spasticity derives from hyperactivity of the stretch reflex caused by damaged corticospinal tract function, ${ }^{6}$ and motor cortex LTP induced by $\mathrm{iTBS}^{7,8}$ alleviates this symptom by increasing the excitability of the corticospinal tract. In this respect, the CB1R has been implicated in LTP in both animals ${ }^{5}$ and humans, as treatment with cannabis in MS favors TBS-induced LTP. ${ }^{6}$ Notably, iTBS-induced effects originate cortically at impinging on pyramidal output cells, ${ }^{26}$ which are also particularly sensitive to CB1R modulation. ${ }^{27}$

It is interesting that the brain-derived neurotrophic factor, an essential player in LTP induction and maintenance, is increased by exercise, ${ }^{28,29}$ and its genetic polymorphism can impair both TBSinduced LTP $^{30}$ and exercise-induced cortical reorganization in humans. ${ }^{31}$

The results of our investigation could be extended to PT effects in patients with diseases other than MS, such as stroke. However, it is also possible that the specific mechanisms of brain damage in MS, which are greatly dependent on the release of inflammatory molecules by infiltrating immune cells, could exacerbate the negative influence of the genetically determined defective CB1R function on synaptic plasticity and motor recovery, as both EAE and MS have been associated with reduced CB1R function. ${ }^{32}$ Indeed, downregulation of CB1R caused by both genetic defect or subchronic tetrahydrocannabinol exposure causes activation of the microglia in mouse cerebellum. This microglia activation causes deficits in cerebellar associative learning and motor coordination mediated by release of proinflammatory cytokines such as interleukin- $1 \beta{ }^{33}$

Accordingly, proinflammatory molecules (namely interleukin-1 $\beta$ ) have been demonstrated to inhibit CB1Rs in mice. ${ }^{34}$ Moreover, other cytokines that can be altered during inflammatory activity, such as the platelet-derived growth factor, have been shown to influence TBS-induced LTP and accumulation of disability in MS. ${ }^{35,36}$

TBS aftereffects can last up to 1 hour $^{37}$; however, in our study the recording was limited to the first 15 minutes only. Moreover, because determination of AMT requires voluntary activation of the target muscle, this may have caused metaplastic interactions with the subsequent iTBS protocol..$^{37}$ Therefore our findings may be limited to early LTP induction and may be related to either iTBSinduced LTP or its metaplastic interaction with prior voluntary activation. Finally, even though PT was administered following a detailed program, exercises differed among patients according to their level of impairment and disability. In addition, the effects of training on ambulation, balance, safety, and range of motion were not measured through specific scales. PT programs usually last several months to produce effective results. ${ }^{18}$ Because our PT program lasted only 2 weeks, we cannot exclude the possibility that long-AATn CNR1 patients may still be responsive to a longer PT program. These limitations may prevent the conclusion that exercise is more beneficial in patients with short-AATn. Further studies are needed to confirm our results and hypothesis and to test the effects of the CNR1 genetic variants both in healthy individuals and in other disease conditions and how they interact with other factors that may potentially influence TBSinduced LTP and effects of exercise.

Our results provide the first evidence that genetic differences within the CB1R may determine at least in part the variable clinical response to PT and strengthen the proposed involvement of CB1Rs in the control of spasticity. This information might be of great relevance for patient stratification and personalized PT. 
Defective plasticity mechanisms in long-AATn CNR1 patients with MS could also account for worse clinical evolution, ${ }^{19,38}$ as plasticity plays a key role in spontaneous compensation of brain damage, $35,36,39$ implying that defective plasticity reserve may contribute to clinical progression. It is interesting that genome-wide association studies did not identify the genetic area containing the $C N R 1$ gene as related to MS susceptibility ${ }^{40}$; however, long-AATn CNRI $(\geq 13)$ alleles are more frequent in patients with primary progressive MS, ${ }^{38}$ a condition characterized by progressive clinical worsening of disability with no periods of remission.

\section{AUTHOR CONTRIBUTIONS}

Dr. Mori: study concept and design, acquisition of data, analysis, interpretation, and manuscript preparation. Dr. Ljoka: study concept and design, acquisition of data. Dr. Nicoletti: acquisition of data. Dr. Kusayanagi: acquisition of data. Dr. Buttari: acquisition of data. Ms. Giordani: acquisition of data. Dr. Rossi: study concept and design. Dr. Foti: study concept and design. Dr. Centonze: study concept and design, interpretation, and manuscript preparation.

\section{STUDY FUNDING}

This study was sponsored by the Fondazione Italiana Sclerosi Multipla (FISM Special Project), by the Italian National Ministero dell'Università, and by Fondazione Baroni to D.C.

\section{DISCLOSURE}

F. Mori received travel funding from Teva Pharmaceuticals and MerckSerono, received speaker honoraria from Merck-Serono, is an Associate Editor for BMC Neurology, and consulted for Grifols Italia. C. Ljoka, C.G. Nicoletti, and H. Kusayanagi report no disclosures. F. Buttari has received speaker honoraria from Novartis and Teva. L. Giordani reports no disclosures. S. Rossi is on the advisory board for Biogen Idec, Teva, and Novartis; has received speaker honoraria from Novartis, Teva, and Biogen Idec; and has received travel funding from Novartis, Teva, Merck-Serono, and SanofiAventis. C. Foti is on the editorial board for Bio Med Center Med Sport. D. Centonze is on the scientific advisory board for Teva Pharmaceutical Industries Ltd, Merck-Serono, Bayer Schering, and Novartis; has received speaker honoraria and travel funding from Sanofi-Aventis, Merck-Serono, Serono Symposia International Foundation, Bayer Schering Pharma, and Biogen-Dompe AG; and has received research support from Merck-Serono, Teva, Novartis, Bayer Schering, Sanofi-Aventis, Biogen Idec, Italian National Ministry of Education, and Foundazione Italiana Sclerosi Multipla (FISM). Go to Neurology.org/nn for full disclosures.

Received June 23, 2014. Accepted in final form October 21, 2014.

\section{REFERENCES}

1. Lin TW, Kuo YM. Exercise benefits brain function: the monoamine connection. Brain Sci 2013;3:39-53.

2. Taubert M, Lohmann G, Margulies DS, Villringer A, Ragert P. Long-term effects of motor training on restingstate networks and underlying brain structure. Neuroimage 2011;57:1492-1498.

3. Chen R, Cohen LG, Hallett M. Nervous system reorganization following injury. Neuroscience 2002;111:761-773.

4. Kesselring J, Beer S. Symptomatic therapy and neurorehabilitation in multiple sclerosis. Lancet Neurol 2005;4: 643-652.

5. Madroñal N, Gruart A, Valverde O, Espadas I, Moratalla R, Delgado-García JM. Involvement of cannabinoid CB1 receptor in associative learning and in hippocampal CA3-CA1 synaptic plasticity. Cereb Cortex 2012; 22:550-566.
6. Koch G, Mori F, Codecà C, et al. Cannabis-based treatment induces polarity-reversing plasticity assessed by theta burst stimulation in humans. Brain Stimul 2009;2:229-233.

7. Mori F, Codecà C, Kusayanagi H, et al. Effects of intermittent theta burst stimulation on spasticity in patients with multiple sclerosis. Eur J Neurol 2010;17:295-300.

8. Mori F, Ljoka C, Magni E, et al. Transcranial magnetic stimulation primes the effects of exercise therapy in multiple sclerosis. J Neurol 2011;258:1281-1287.

9. Novotna A, Mares J, Ratcliffe S, et al. Sativex Spasticity Study Group. A randomized, double-blind, placebo-controlled, parallel-group, enriched-design study of nabiximols* (Sativex $\left.\left({ }^{\circledR}\right)\right)$, as add-on therapy, in subjects with refractory spasticity caused by multiple sclerosis. Eur J Neurol 2011; 18:1122-1131.

10. De Chiara V, Errico F, Musella A, et al. Voluntary exercise and sucrose consumption enhance cannabinoid CB1 receptor sensitivity in the striatum. Neuropsychopharmacology 2010;35:374-387.

11. Rossi S, Furlan R, De Chiara V, et al. Exercise attenuates the clinical, synaptic and dendritic abnormalities of experimental autoimmune encephalomyelitis. Neurobiol Dis 2009;36:51-59.

12. Zhang PW, Ishiguro H, Ohtsuki T, et al. Human cannabinoid receptor 1: 5' exons, candidate regulatory regions, polymorphisms, haplotypes and association with polysubstance abuse. Mol Psychiatry 2004;9:916-931.

13. Rossi S, Bozzali M, Bari M, et al. Association between a genetic variant of type-1 cannabinoid receptor and inflammatory neurodegeneration in multiple sclerosis. PLoS One 2013;8:e82848. doi: 10.1371/journal.pone.0082848.

14. Polman CH, Reingold SC, Banwell B, et al. Diagnostic criteria for multiple sclerosis: 2010 revisions to the McDonald criteria. Ann Neurol 2011;69:292-302.

15. Kujirai T, Caramia MD, Rothwell JC, et al. Corticocortical inhibition in human motor cortex. J Physiol 1993; 471:501-519.

16. Hanajima R, Ugawa Y, Terao Y, et al. Mechanisms of intracortical I-wave facilitation elicited with paired-pulse magnetic stimulation in humans. J Physiol 2002;538: 253-261.

17. Valls-Solé J, Pascual-Leone A, Wassermann EM, Hallett M. Human motor evoked responses to paired transcranial magnetic stimuli. Electroencephalogr Clin Neurophysiol 1992;85:355-364.

18. White LJ, Dressendorfer RH. Exercise and multiple sclerosis. Sports Med 2004;34:1077-1100.

19. Rossi S, Buttari F, Studer V, et al. The (AAT)n repeat of the cannabinoid CB1 receptor gene influences disease progression in relapsing multiple sclerosis. Mult Scler 2011; 17:281-288.

20. De Roo M, Klauser P, Garcia PM, Poglia L, Muller D. Spine dynamics and synapse remodeling during LTP and memory processes. Prog Brain Res 2008;169:199-207.

21. Tyc F, Boyadjian A, Devanne H. Motor cortex plasticity induced by extensive training revealed by transcranial magnetic stimulation in human. Eur J Neurosci 2005;21:259-266.

22. Raichlen DA, Foster AD, Seillier A, Giuffrida A, Gerdeman GL. Exercise-induced endocannabinoid signaling is modulated by intensity. Eur J Appl Physiol 2013; 113:869-875.

23. Dubreucq S, Durand A, Matias I, et al. Ventral tegmental area cannabinoid type- 1 receptors control voluntary exercise performance. Biol Psychiatry 2013;73:895-903. 
24. Baker D, Pryce G, Croxford JL, et al. Cannabinoids control spasticity and tremor in a multiple sclerosis model. Nature 2000;404:84-87.

25. Koppel BS, Brust JC, Fife T, et al. Systematic review: efficacy and safety of medical marijuana in selected neurologic disorders: report of the Guideline Development Subcommittee of the American Academy of Neurology. Neurology 2014;82:1556-1563.

26. Di Lazzaro V, Pilato F, Saturno E, et al. Theta-burst repetitive transcranial magnetic stimulation suppresses specific excitatory circuits in the human motor cortex. J Physiol 2005;565:945-950.

27. Auclair N, Otani S, Soubrie P, Crepel F. Cannabinoids modulate synaptic strength and plasticity at glutamatergic synapses of rat prefrontal cortex pyramidal neurons. J Neurophysiol 2000;83:3287-3293.

28. Liu HL, Zhao G, Cai K, Zhao HH, Shi LD. Treadmill exercise prevents decline in spatial learning and memory in APP/PS1 transgenic mice through improvement of hippocampal longterm potentiation. Behav Brain Res 2011;218:308-314.

29. Coelho FG, Gobbi S, Andreatto CA, Corazza DI, Pedroso RV, Santos-Galduróz RF. Physical exercise modulates peripheral levels of brain-derived neurotrophic factor (BDNF): a systematic review of experimental studies in the elderly. Arch Gerontol Geriatr 2013;56:10-15.

30. Cheeran B, Talelli P, Mori F, et al. A common polymorphism in the brain-derived neurotrophic factor gene (BDNF) modulates human cortical plasticity and the response to rTMS. J Physiol 2008;586:5717-5725.

31. Kleim JA, Chan S, Pringle E, et al. BDNF val66met polymorphism is associated with modified experience-dependent plasticity in human motor cortex. Nat Neurosci 2006;9:735-737.
32. Centonze D, Bari M, Rossi S, et al. The endocannabinoid system is dysregulated in multiple sclerosis and in experimental autoimmune encephalomyelitis. Brain 2007;130: 2543-2553.

33. Cutando L, Busquets-Garcia A, Puighermanal E, et al. Microglial activation underlies cerebellar deficits produced by repeated cannabis exposure. J Clin Invest 2013;123: 2816-2831.

34. Rossi S, Sacchetti L, Napolitano F, et al. Interleukin- $1 \beta$ causes anxiety by interacting with the endocannabinoid system. J Neurosci 2012;32:13896-13905.

35. Mori F, Rossi S, Piccinin S, et al. Synaptic plasticity and PDGF signaling defects underlie clinical progression in multiple sclerosis. J Neurosci 2013;33:19112-19119.

36. Mori F, Nicoletti CG, Rossi $S$, et al. Growth factors and synaptic plasticity in relapsing-remitting multiple sclerosis. Neuromolecular Med 2014;16:490-498.

37. Goldsworthy MR, Pitcher JB, Ridding MC. The application of spaced theta burst protocols induces long-lasting neuroplastic changes in the human motor cortex. Eur J Neurosci 2012;35:125-134.

38. Ramil E, Sánchez AJ, González-Pérez P, et al. The cannabinoid receptor 1 gene (CNR1) and multiple sclerosis: an association study in two case-control groups from Spain. Mult Scler 2010;16:139-146.

39. Mori F, Kusayanagi H, Nicoletti CG, Weiss S, Marciani MG, Centonze D. Cortical plasticity predicts recovery from relapse in multiple sclerosis. Mult Scler 2014;20:451-457.

40. Oksenberg JR, Baranzini SE, Sawcer S, Hauser SL. The genetics of multiple sclerosis: SNPs to pathways to pathogenesis. Nat Rev Genet 2008;9:516-526. 


\section{Neurology \\ Neuroimmunology \& Neuroinflammation}

\section{CB1 receptor affects cortical plasticity and response to physiotherapy in multiple}

sclerosis

Francesco Mori, Concetta Ljoka, Carolina G. Nicoletti, et al.

Neurol Neuroimmunol Neuroinflamm 2014;1;

DOI 10.1212/NXI.0000000000000048

This information is current as of December 11, 2014

\section{Updated Information \& \\ Services}

References

Permissions \& Licensing

Reprints including high resolution figures, can be found at:

http://nn.neurology.org/content/1/4/e48.full.html

This article cites 40 articles, 2 of which you can access for free at: http://nn.neurology.org/content/1/4/e48.full.html\#\#ref-list-1

Information about reproducing this article in parts (figures,tables) or in its entirety can be found online at:

http://nn.neurology.org/misc/about.xhtml\#permissions

Information about ordering reprints can be found online: http://nn.neurology.org/misc/addir.xhtml\#reprintsus

Neurol Neuroimmunol Neuroinflamm is an official journal of the American Academy of Neurology.

Published since April 2014, it is an open-access, online-only, continuous publication journal. Copyright $\odot$ 2014 American Academy of Neurology. All rights reserved. Online ISSN: 2332-7812.

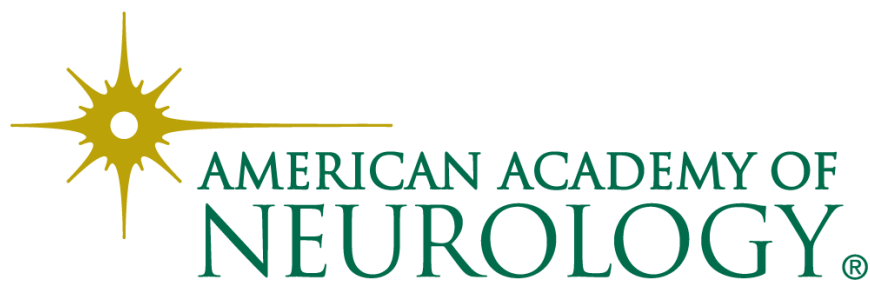

